\title{
Meta-Analysis of Heuristic Approaches for Optimizing Node Localization and Energy Efficiency in Wireless Sensor Networks
}

\author{
Oluwasegun J. Aroba, Nalindren Naicker, Timothy T. Adeliyi, Ropo E. Ogunsakin
}

\begin{abstract}
Background: In the literature node localization and energy efficiency are intrinsic problems often experienced in wireless sensor networks (WSNs). Consequently, various heuristic approaches have been proposed to allay the challenges faced by WSNs. However, there is little to nothing in the literature to support which of the heuristic approaches is best in optimizing node localization and energy efficiency problems in WSN. The aim of this paper is to assess the best heuristic approach to date on resolving the node localization and energy efficiency in WSNs. Method: The extraction of the relevant articles was designed following the technique of preferred reporting items for systematic reviews and meta-analyses (PRISMA). All the included research articles were searched from the widely used databases of Google Scholar and Web of Science. All statistical analysis was performed with the fixed-effects model and the random-effects model implementation in RStudio. The overall pooled global estimate and categorization of performance for the heuristic approaches were presented in forest plots. Results: A total of 18 studies were included in this meta-analysis and the overall pooled estimated categorization of the heuristic approaches was 35\% (95\% CI $(13 \%, 67 \%))$. According to subgroup analysis the pooled estimation of heuristic approach with hyper-heuristic was $71 \%$ (95\% CI: 6\% to 99\%), I2 = 100\%) while the hybrid heuristic, was $31 \%(95 \%$ CI: $3 \%$ to $87 \%, I 2=100 \%)$ and metaheuristic was 21\%(95\% CI: $9 \%$ to $41 \%$, I2 = 100\%). Conclusion: It can be concluded based on the experimental results that hyper-heuristic approach outclassed the hybrid heuristic and metaheuristic approaches in optimizing node localization and energy efficiency in WSNs.
\end{abstract}

Keywords: Hyper-Heuristic, Hybrid Heuristic, Metaheuristic, Node Localization, Wireless Sensor Network.

\section{INTRODUCTION}

Wireless sensor networks (WSN) is a key component in the emerging Internet-of-Things (IoT) paradigm. The last decade has seen the development of wireless sensor networks with a multitude of applications built around the sensors [1]. Wireless sensor networks are self-arrangement of the network system which consists of many micro nodes with a low-cost component of data processing and wireless communication [2].

Revised Manuscript Received on September 15, 2020.

* Correspondence Author

Oluwasegun. J. Aroba*, Department of Information Systems. A graduate of Information Technology University from prestigious Coventry University United Kingdom,

Dr. N. Naicker Department of Information Systems; Information Technology and Computer Science.

Dr. Timothy T. Adeliyi Department of Information Technology at the Durban University of Technology.

Dr. R. E. Ogunsakin, Department of Information Technology at the Durban University

(C) The Authors. Published by Blue Eyes Intelligence Engineering and Sciences Publication (BEIESP). This is an open access article under the CC BY-NC-ND license (http://creativecommons.org/licenses/by-nc-nd/4.0/)
Wireless sensor networks make use of node localization with efficient energy consumption and is extensively used to identify the current location of the sensor nodes [3]. WSNs with node localization and energy efficiency have uses in many different sectors such as telecommunications, energy companies and health care organizations across the globe with specific applications in area monitoring, energy saving capacity, health care monitoring, machine health monitoring, data logging, threat detection, landslide monitoring and uses within educational institutions. In WSNs the nodes form a network through self-organization [3]. WSNs can retrieve, collect and process the monitored data with the synergy of localization and the energy transmission in the network and send it to the user terminal. WSN nodes are powered by a battery that has limited energy. It is inconvenient for the battery to be recharged when in operation for efficiency reasons which leads to poor operational functionality in WSNs when the node dies. The life-time of the sensor node depends to a large extent on the charged battery life, and the unconsidered energy consumption will cause the network to depreciate prematurely and reduce the network lifetime. WSN is an emerging paradigm of computing and networking, which can be defined as a network of minuscule, diminutive, inexpensive, and keenly intellective devices called sensor nodes [4]. WSN is integrated into other types of network environments such as IoT, for example, WSNs use temperature, sound, and pressure sensors to monitor the status of the environment. Lots of research work has been delved into in wireless sensor networks. Ant Colony was proposed to get knowledge of the sensor node localization for applications of the WSNs for an optimal critical path of data transmission [3]. Similarly, the Elephant Herding Optimization algorithm was used for tackling the node localization problem in WSN [6]. Also, the Flower Pollination Algorithm strategy was deployed for optimization localization issues in WSNs [3]. Node Localization problems in WSNs is one of the most significant headaches in the domain for geographical co-ordinates within sensor node with the Elephant Herding Optimization algorithm (EHO) being the chosen technique used to meet challenges [5]. In the period starting from 2016 to 2019, various scholars have agreed that there have been challenges facing wireless sensor network [3-5]. Since WSN have a high practical value, many scholars have conducted in-depth research in this area. Wireless sensor network energy for node localization consumption during data transmission needs to be reduced. Various techniques are available in the literature to solve this problem that focused mainly on node localization energy efficiency

\section{Published By:}

Blue Eyes Intelligence Engineering and Sciences Publication

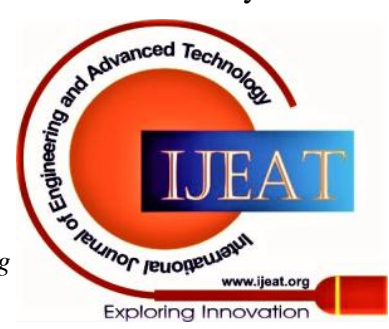




\section{Meta-Analysis of Heuristic Approaches for Optimizing Node Localization and Energy Efficiency in Wireless Sensor Networks}

and paid less attention to other issues such as quality of service, coverage, transmission and detection of connectivity. Some of the protocols to validate the proposed analysis of Metaheuristic, Hyper-heuristics and Hybrid heuristic on the selection of optimal paths face challenges related to the shortest path that offers the highest throughput and smallest delay to the destination to enhance the lifespan of a wireless sensor network. Achieving this by optimizing the clustering, improving node localization, optimizing cluster head selection, and using energy-efficient routing by considering localization parameters is a challenge and motivation for future research.

\section{RELATED WORK}

Different approaches on how energy efficiency and node localization have impacted the operation of sensor nodes not delivering the packet to the sink node are evident from the literature. We present the literature from the three major heuristic approaches, namely, Meta-heuristic, Hybrid Heuristic and Hyper-heuristic in this section.

\section{Meta-Heuristic Method}

Meta-heuristic methods use a combination of two or more algorithms which some of the researchers have used with their various algorithmic approaches as mentioned in this research work to solve problems of sensor nodes. It is considered a higher-level procedure that presents solutions to optimization problems in wireless sensor networks. The shortcomings of meta-heuristic methodologies are high energy consumption and consequently short network life-time, which is the main reason that restricts its development. At present, inspired by the characteristics of wireless sensor networks, a variety of routing protocols have been proposed. The routing protocols are mainly divided into categories in terms of network topology [7]. One of the well-known life-time issues of WSN is that the set of sensors that are elected as the clustering heads run out of their energy faster [8]. This problem is known as clustering head election problem (CHEP). The medium to implement a clustering algorithm called low-energy adaptive clustering hierarchy (LEACH) was presented to solve this problem, which has become a popular clustering method [9]. There was a tendency to attribute the success of LEACH to its original idea of avoiding any sensor from being elected as the cluster head $(\mathrm{CH})$ more frequently than the other sensors. In other words, LEACH will not elect the same sensor as a $\mathrm{CH}$ every time, and all the sensors have a chance to be elected as a $\mathrm{CH}$. According to the $\mathrm{CH}$ election rule of $\mathrm{LEACH}$, it is expected that every sensor has the same chance to be elected as the $\mathrm{CH}$ by a fair mechanism. For this reason, by using $\mathrm{LEACH}$, a sensor will run out of its energy quickly even though it has been elected as a $\mathrm{CH}$ [9].

Most studies have focused on metaheuristics approaches to resolve one of the following problems in WSN, namely, consumption, energy efficiency, clustering of sensors nodes which leads to delay of packets, data communication from source to destination due to its clustering-based information gathering. These studies are proposed in reviews of works of literature in [10-16]. The use of metaheuristic approaches algorithms to optimize energy usage in WSN, the efficiency of energy and clustering with the use of the Bat algorithm to solve non-linear benchmark design for convergence rate was proposed [17]. Galaxy- Based Search algorithm was implemented for the energy efficiency. The Intelligent Water
Drops algorithm, new swarm-based optimization algorithm inspired from observing natural water drops that flow in rivers were tested with some knapsack problems and optimal solutions were obtained [18]. The meta-heuristic clustering design problem is another issue mentioned with the use of a Teaching Learning-Based Optimization algorithm to find the centroids to specified clusters performance technique that will help in resolving clustering problems in WSN [17].

Another perspective of Meta-heuristics approaches in journals papers concentrated on the localization issues in WSNs that were delved into with the use of novel Artificial Swarm Intelligence algorithms. In order to form prediction ability, the Krill Herd algorithm was deployed for optimizing localization for a wide range of optimization problems to outperform the existing algorithms. Another meta-heuristic bio-inspired optimization algorithm, the Cuttlefish Optimization algorithm was also explored [19].

Other Meta-heuristic algorithms proposed in solutions for routing, localization deficiency in nodes, energy consumption and clustering are as follows: Colliding Bodies Optimization algorithm [20], Mass and Energy Balance algorithm, Harris Hawk Optimization [21], Emperor Penguin Optimization algorithm [22], Momentum Balance algorithm [22], Memetic algorithm [23]. Similarly, there seems to be no concrete solutions and there exist gaps and there is still no general agreement on the Meta-heuristic approach although there has been the further investigation and new methods are being proposed by researchers from 2009 to date.

\section{Hybrid Heuristic Approach}

Furthermore, a strategy to strengthen the hyper-heuristic has been to hybridize which is seen in the journals. The combination of different heuristics, Meta-heuristic, Sim-heuristic, Hyper-heuristic is brought together proffer solutions to problems [38-39]. The recent hybridization of computer science research diverted into hybridization for specifying intentions where the metaheuristics machine learning algorithm improved analysis [40-41]. Until now Hybrid Heuristic approaches were used in recent publications for looking into task scheduling to minimize the delay and optimize the nodes energy consumption during packet delivery in the WSN. Hybrid algorithms help to combine Particle Swarm algorithm and the Ant Colony optimization algorithms to produce optimal solutions to WSN problems [42-43].

Thus far the introduction of computing in the intelligent manufacturing environment adopted the embryo of virtual technology through task scheduling in WSNs. Moreover, a Hybrid Heuristic algorithm for application to the multidimensional knapsack problem and Binarization with $\mathrm{dB}$-scan were improved for both the best and the average sensors nodes with possible directions for further extension and improvements [44,55].

\section{Hyper-Heuristic Approach}

Hyper-heuristic methodology approaches were further envisaged in the literature. It is known that the word Hyper-heuristic was first explored in 1997 to show the protocols that combine artificial intelligence methods in the context of automated theorem explanation for WSN [24]. Similarly,

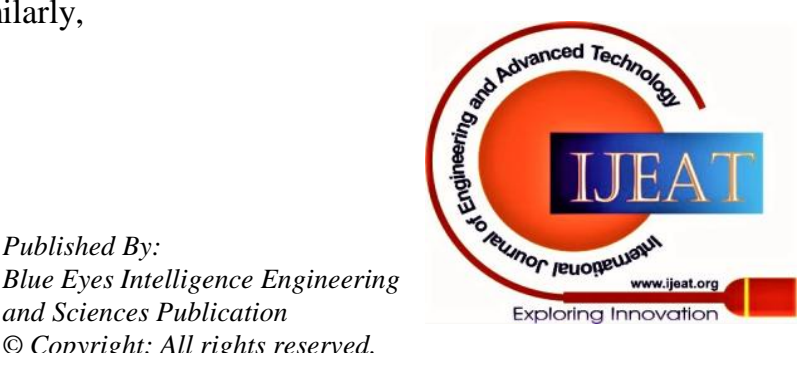


Hyper-heuristic has been mentioned again in the 2000s to choose a heuristic in the combinatorial optimization resolution for a high-level approach [25-29].

A Hyper-heuristic is a process of automation methodology for selecting or producing heuristics to generate hard computational search problems [29].

Hyper-heuristic research consists of different approaches that distribute the common goal of automating the adaptation and design of heuristic methods to solve hard computational search issues [26,30]. We are raising the level of generality for search methodologies on comparative analysis.

With regards to the most result of Hyper-heuristic algorithms in the literature shows that they can propose a better result than the other algorithms in solving localization optimization problems using a smart guess or strategies. The table below outline's the studies conducted on Metaheuristic, Hyper-heuristic and hybrid heuristic approaches from the extant of literature.

\begin{tabular}{|c|c|c|c|c|c|}
\hline Authors & Approach & Algorithm & $\begin{array}{l}\text { No. of } \\
\text { Sensors Node }\end{array}$ & $\begin{array}{l}\text { Number of } \\
\text { Rounds }\end{array}$ & Problem Focused \\
\hline [45] & Metaheuristic & $\begin{array}{l}\text { Robust Harmony } \\
\text { Search Algorithm }\end{array}$ & 1863 & 3000 & $\begin{array}{l}\text { Data aggregation and } \\
\text { balancing energy }\end{array}$ \\
\hline [46] & Metaheuristic & $\begin{array}{l}\text { H-Best Particle } \\
\text { Swarm Optimization } \\
\text { (HPSO) }\end{array}$ & 120 & 40 & Node localization \\
\hline [47] & Metaheuristic & $\begin{array}{l}\text { Improved } \\
\text { Metaheuristic }\end{array}$ & 700 & 100 & $\begin{array}{l}\text { Energy Efficiency \& } \\
\text { Clustering }\end{array}$ \\
\hline [48] & Metaheuristic & $\begin{array}{l}\text { Multi Heuristic } \\
\text { Energy Efficiency } \\
\text { Search Data } \\
\text { Aggregation } \\
\text { Algorithm } \\
\text { (MH-EESDA) }\end{array}$ & 100 & 400 & Energy efficiency \\
\hline [49] & Metaheuristic & Metaheuristic & 100 & 2500 & Energy prolong lifetime \\
\hline$[50]$ & Metaheuristic & $\begin{array}{l}\text { Distributed } \\
\text { Metaheuristic }\end{array}$ & $80-100$ & 50 & Node Localization \\
\hline [51] & Metaheuristic & $\begin{array}{l}\text { ARSH-FATI -CH } \\
\text { Algorithm }\end{array}$ & $100-400$ & $1000-8000$ & Depletion of Lifetime energy \\
\hline [36] & Hyper-heuristic & $\begin{array}{l}\text { High-performance } \\
\text { meta-heuristic } \\
\text { Algorithm with } \\
\text { LEACH }\end{array}$ & 1000 & 3000 & Energy Consumption \\
\hline [40] & Hyper-heuristic & $\begin{array}{l}\text { Energy-aware } \\
\text { computing } \\
\text { framework algorithm }\end{array}$ & 120 & 340 & $\begin{array}{l}\text { Energy-aware Osmotic } \\
\text { Framework management }\end{array}$ \\
\hline [32] & Hyper-heuristic & $\begin{array}{l}\text { Unified Heuristic Bat } \\
\text { Algorithm (UHBA) }\end{array}$ & 100 & 2000 & $\begin{array}{l}\text { Energy consumption and } \\
\text { Cluster head }\end{array}$ \\
\hline [52] & Hyper-heuristic & $\begin{array}{l}\text { Panacea -no collision } \\
\text { detection (NCD) } \\
\text { Algorithm }\end{array}$ & 1000 & $100-1000$ & $\begin{array}{l}\text { Low latency and energy } \\
\text { efficiency }\end{array}$ \\
\hline [53] & Hyper-heuristic & $\begin{array}{l}\text { Simulated Annealing } \\
\text { (SA) and Variable } \\
\text { Neighbourhood } \\
\text { Search (VNS) }\end{array}$ & 100 & 190 & Energy Efficiency \\
\hline
\end{tabular}

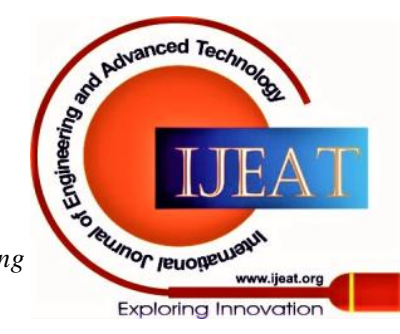


Meta-Analysis of Heuristic Approaches for Optimizing Node Localization and Energy Efficiency in Wireless Sensor Networks

\begin{tabular}{|l|l|l|l|l|l|}
\hline Authors & Approach & Algorithm & $\begin{array}{l}\text { No. of } \\
\text { Sensors Node }\end{array}$ & $\begin{array}{l}\text { Number of } \\
\text { Rounds }\end{array}$ & Problem Focused \\
\hline$[54]$ & Hybrid & $\begin{array}{l}\text { Leach and Monkey } \\
\text { Search Algorithm }\end{array}$ & 100 & 1800 & $\begin{array}{l}\text { Limited battery Power in } \\
\text { LEACH }\end{array}$ \\
\hline$[56]$ & Hybrid & $\begin{array}{l}\text { Energy Center Search } \\
\text { using Particle Swarm } \\
\text { Optimization } \\
\text { (EC-PSO) }\end{array}$ & 400 & 1400 & Clustering and Energy \\
\hline$[57]$ & Hybrid & $\begin{array}{l}\text { Hyperelliptic Curve } \\
\text { Cryptography } \\
\text { (HECC) }\end{array}$ & 100 & 100 & $\begin{array}{l}\text { Energy Efficiency and } \\
\text { Routing }\end{array}$ \\
\hline$[58]$ & Hybrid & $\begin{array}{l}\text { Clustering and } \\
\text { Tree-based Routing } \\
\text { Protocols }\end{array}$ & 100 & 7000 & Energy Efficiency \\
\hline$[59]$ & Hybrid & 500 & 3500 & $\begin{array}{l}\text { Cluster Head based } \\
\text { Energy-aware }\end{array}$ \\
\hline$[60]$ & Hybrid & $\begin{array}{l}\text { Hybrid Memetic } \\
\text { Framework }\end{array}$ & 100 & $80-300$ & $\begin{array}{l}\text { Energy consumption and } \\
\text { network coverage loss }\end{array}$ \\
\hline
\end{tabular}

Table 1: Studies conducted on Meta-heuristic, Hybrid Heuristic and Hyper-Heuristic Approaches

\section{MATERIALS AND METHODS}

\section{Literature Strategy}

This study was carried out with the Preferred Reporting Item for System Reviews and Meta-Analyses (PRISMA) [61-62]. PRISMA provided a checklist of the recommended items to address during the systematics review. The systematic review considered article searches from Google Scholar and Web of Science databases and scrutinizing relevant articles [63]. The PRISMA helped us to streamline more than 306 citations, journals, patents that could be found on metaheuristics, hyper-heuristics, and hybrid heuristics in wireless sensor networks (Figure 1). The articles from the databases between 2010 and 2020 were considered and identified as shown in Figure 1. As an evidence procedure, a reference list of relevant literature studies was scrutinized manually for citations missed by the electronic database search.

\section{Inclusion Criteria}

The inclusion criteria of the articles focused on literature that was conducted on heuristic approaches highlighting the number of sensor nodes used, the number of rounds (dead node) that addresses node localization, and energy efficiency problems in WSN.

\section{Exclusion Criteria}

The exclusion criteria entail articles written in other languages aside from English, published articles before January 2010 with some study design such as editorial, review, letters to editors, expert opinions, commentaries, blogs, books, books chapters, editorials brief reports, unpublished articles, and thesis were all excluded while selecting the inclusion criteria.

\section{Quality Assessment}

The information that was selected to be part of the selection inclusion criteria were extracted from the various studies. The study with $100 \%$ information that fit into the methods of selection of inclusion criteria was appropriate for the systematic review and meta-analysis of the heuristic approaches.

\section{Statistical data analysis}

In preparation for the statistical analysis, the extracted data was entered into an excel spreadsheet. Both the fixed-effects model and the random-effects model were used for the meta-analysis while the heterogeneity among the extracted approaches was established using the $\mathrm{I}^{2}$ statistic [64-65]. Also, heterogeneity was employed to comprehend the understanding between the random-effects model against the fixed-effects model in a meta-analysis. In the case of statistical significance in heterogeneity, this study steps further by conducting a subgroup analysis to establish where the significance lies. In the case of publication bias, we deployed Egger and Begg's tests. Because of the nature of the heuristic approaches considered in this study, all original data included in the analysis were first transformed by a double arcsine method before performing a meta-analysis of metaheuristics, hyper-heuristics, and hybrid heuristics methodology. The essence of this transformation is to make them conform to normal distribution. All the statistical analysis was done in RStudio software.

Figure 1 below shows the flow diagram of the database searches using PRISMA

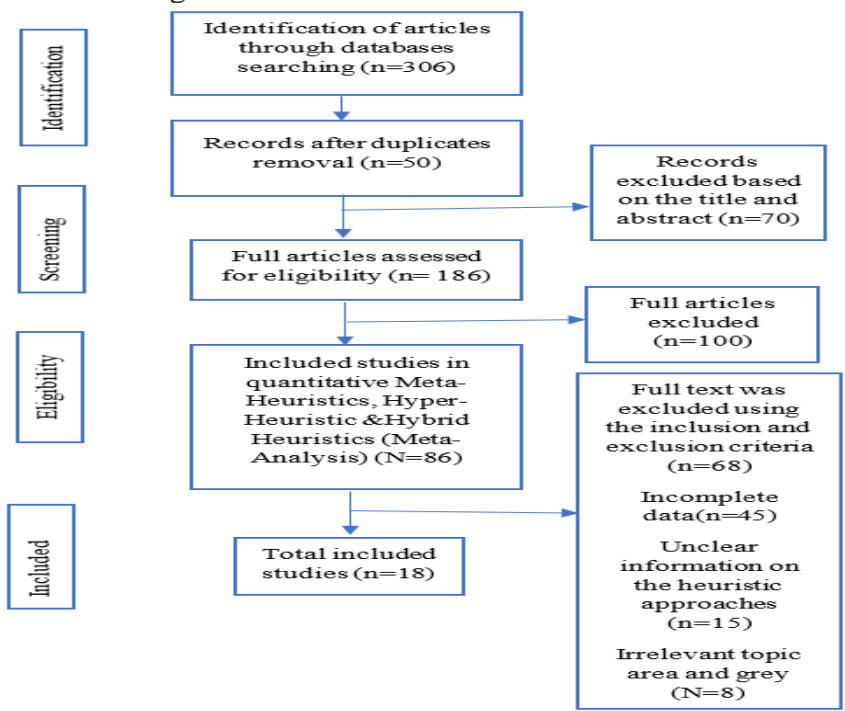

Figure 1. Flow diagram of the database searches (Preferred Reporting Items for Systematic Reviews and Meta-Analysis)

Published By:

Blue Eyes Intelligence Engineering and Sciences Publication

(C) Convriaht: All riahts reserved. 


\section{RESULTS}

The overall pooled estimate using the fixed-effect model showed significant heterogeneity between the studies. Thus, we performed analysis using the random-effects model. Using the random-effects model, the overall estimated pooled of the various heuristic approaches reported by the 18 studies was $35 \%(95 \%$ CI $(13 \%, 67 \%))$ with $\left(I^{2}=100 \%\right)$. The pooled estimation of heuristic approaches is presented using the forest plot as shown in Figure 2.

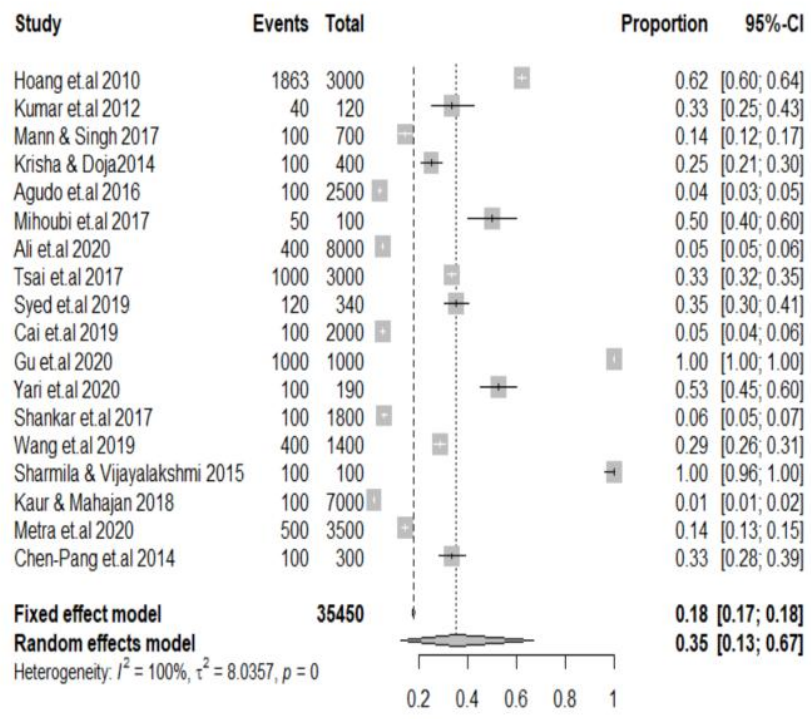

Figure 2. Forest plot of the global estimate of various heuristic approaches.

Subgroup analysis by categorization of the hybrid was conducted to assess the potential heterogeneity between studies. Of the 18 studies, the highest estimated found in studies conducted with hyper-heuristic approach was (71\% (95\% CI: $6 \%$ to $99 \%$ ), $\mathrm{I}^{2}=100 \%$ ), followed by studies conducted with hybrid heuristic approach, was 31\% (95\% CI: $3 \%$ to $87 \%$ ), $\mathrm{I}^{2}=100 \%$ and metaheuristic approach was 21\%(95\% CI: $9 \%$ to $\left.41 \%, \mathrm{I}^{2}=100 \%\right)$. These statistics are shown in Figure 3 below.

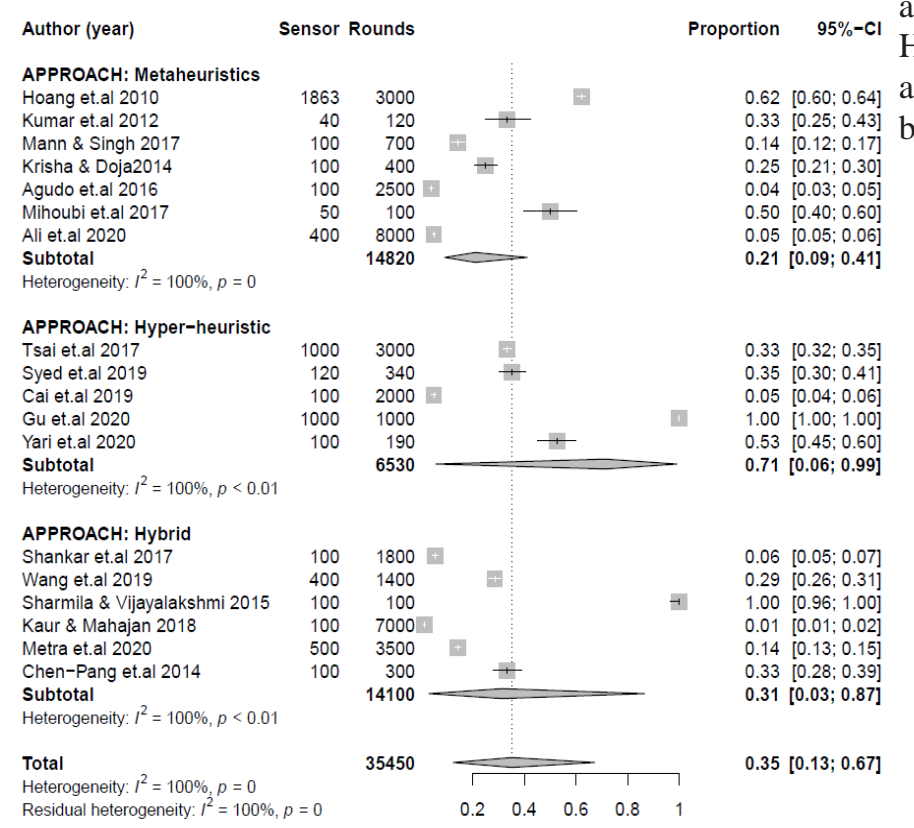

Figure 3. Subgroup analysis by categorization on the performance of the heuristic approaches
Table 2 displays the meta-regression model to assess the source of heterogeneity.

Table 2: Meta-regression model to assess the source of heterogeneity

\begin{tabular}{|l|l|l|l|l|}
\hline $\begin{array}{l}\text { Sources of } \\
\text { heterogeneity }\end{array}$ & Estimates & $\begin{array}{l}\text { Standard } \\
\text { error }\end{array}$ & 95\% CI & P-value \\
\hline $\begin{array}{l}\text { Year of } \\
\text { publication }\end{array}$ & 0.0012 & 0.1868 & $\begin{array}{c}(-0.3649 \\
, 0.3672)\end{array}$ & 0.9951 \\
\hline Sample size & 0.0018 & 0.0011 & $\begin{array}{l}-0.0005, \\
0.0040)\end{array}$ & 0.1201 \\
\hline
\end{tabular}

Due to variations in sample size, studies, inclusion criteria, and methodology utilized, heterogeneity in meta-analysis becomes inevitable. The value of $\mathrm{I}^{2}$ in this meta-analysis is high and that necessitated the use of a random effect model to adjust for the observed variability. Besides, there is still the presence of heterogeneity in the sub-group analysis. Thus, we employed meta-regression to investigate further the sources of heterogeneity by using the year of publication and sensors node as covariates. Based on the previous finding, meta-regression is preferable to the sub-group method of analysis for investigating heterogeneity, since it allows the assessment of more than one covariate concurrently. Based on the result of the meta-regression model, both years of publication sensors node were not statistically significant for the presence of heterogeneity (Table 2). Hence, we can affirm that heterogeneity is due to the significant difference in the sensor's node. Publication bias was further assessed via Begg and Egger's tests, of which the possibility of publication bias was assessed using the Duval and Tweedie Trim and Fill method. This approach works by removing the smallest studies and recalculating the overall effect size until there is symmetry. The method, a rank-based data augmentation technique, formalizes the use of funnel plots, recalculating the overall effect size and outcomes of missing studies, and corrects the meta-analysis to incorporate the theoretical missing studies until there is symmetry. Besides, findings in Figure 4 also attest to the publication bias. Based on the Trim and Fill method, there was evidence of publication bias among the studies, however, its effect was not significant. Hence, we applied the Duval and Tweedie Trim and Fill analysis in the random-effects model to fix the publication bias.

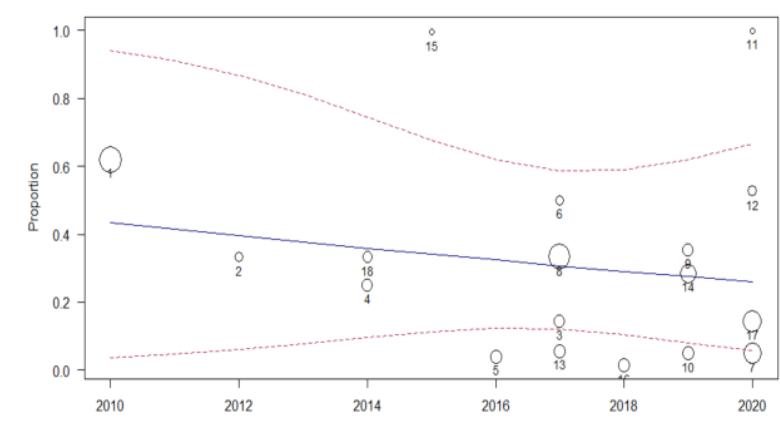

Figure 4. Meta-regression based on year of publication

Published By:

Blue Eyes Intelligence Engineering and Sciences Publication

(C) Copyright: All rights reserved.

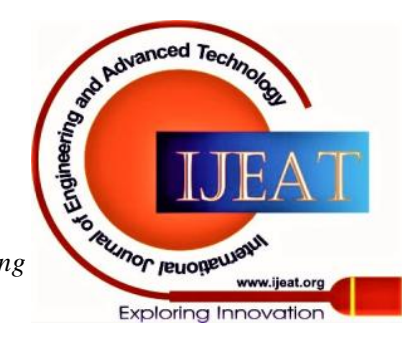




\section{Meta-Analysis of Heuristic Approaches for Optimizing Node Localization and Energy Efficiency in Wireless Sensor Networks}

\section{CONCLUSION}

Node localization and energy efficiency are pertinent problems ravaging wireless sensor network performance. This problem was consistent across many kinds of literature reviewed and the three major approaches used in combating this problem was put into consideration. This study analyzed different heuristic algorithms based on the three major heuristic approaches - Metaheuristic, Hyper-heuristic, and Hybrid heuristic used in optimizing the node localization and energy efficiency problem in WSN. The meta-analysis experimental result divulged that studies that used the Hyper-heuristic approach gave the best result in optimizing the node localization and energy efficiency problem hence increasing the WSN performance. Nevertheless, it does not mean Metaheuristic and Hybrid heuristic are not efficient, but the analysis proved that Hyper-heuristic performance outperforms the metaheuristic and Hybrid heuristic approaches in optimizing the node localization and energy efficiency problems in WSN.

\section{REFERENCES}

1. Arora, S. and Singh, S., 2017. Node localization in wireless sensor networks using butterfly optimization algorithm. Arabian Journal for Science and Engineering, 42(8), pp.3325-3335.

2. Yong-jun, C.H.E.N., and Shen-fang, Y.U.A.N., 2012. Minimum energy consumption topology control for wireless sensor networks. 电 子科技大学学报, 41(4), pp.568-573.

3. Sun, Y., Dong, W., and Chen, Y., 2017. An improved routing algorithm based on ant colony optimization in wireless sensor networks. IEEE Communications Letters, 21(6), pp.1317-1320.

4. Akyildiz, I.F. and Vuran, M.C., 2010. Wireless sensor networks (Vol. 4). John Wiley \& Sons.

5. Strumberger, I., Beko, M., Tuba, M., Minovic, M. and Bacanin, N., 2018, May. Elephant herding optimization algorithm for wireless sensor network localization problem. In Doctoral Conference on Computing, Electrical and Industrial Systems (pp. 175-184). Springer, Cham.

6. Strumberger, I., Minovic, M., Tuba, M. and Bacanin, N., 2019. Performance of elephant herding optimization and tree growth algorithm adapted for node localization in wireless sensor networks. Sensors, 19(11), p.2515.

7. Singh, R. and Verma, A.K., 2017. Energy efficient cross layer based adaptive threshold routing protocol for WSN. AEU-International Journal of Electronics and Communications, 72, pp.166-173.

8. Heinzelman W. R., Chandrakasan A., and Balakrishnan H. 2010. "Energy-efficient communication protocol for wireless microsensor networks." Proceedings of the 33rd Annual Hawaii International Conference on System Sciences. pp 1-10

9. Hoang D, Yadav P, Kumar R, Panda S.K, 2010; A robust harmony search algorithm-based clustering protocol for wireless sensor networks. In: Proceedings of IEEE international conference on communications workshops, pp 1-5

10. Kuila P., and Jana P. K. 2014; "A novel differential evolution-based clustering algorithm for wireless sensor networks." Applied Soft Computing 25:414-425.

11. Jung, H.J., Song, Y., Hong, S.K., Yang, C.H., Hwang, S.J., Jeong, S.Y. and Sung, T.H., 2015. Design and optimization of piezoelectric impact-based micro wind energy harvester for wireless sensor network. Sensors and Actuators A: Physical, 222, pp.314-321.

12. Rao P. C. S., Jana P. K., and Banka H. 2016. "A particle swarm optimization-based energy-efficient cluster head selection algorithm for wireless sensor networks." Wireless Networks 23(7): 2005-2020.

13. Yuan X., Elhoseny M., El-Minor H. K., Riad A.M. 2017 “A Genetic Algorithm-Based, Dynamic Clustering Method Towards Improved C Longevity" Journal of Network and Systems Management Volume 25, Issue 1:21-46.

14. Bozorgi, S. M., and Bidgoli, A. M. 2018. "HEEC: a hybrid unequal energy-efficient clustering for wireless sensor networks." Wireless Networks:1-22.
15. Kaur T., and Kumar D. 2018. "Particle Swarm Optimization-Based Unequal and Fault-Tolerant Clustering Protocol for Wireless Sensor Networks.” IEEE Sensors Journal 18(11): 4614-4622.

16. Mhatre M., Kumar A., and Jha C. K. 2019. "Energy-Efficient Wireless Sensor's Routing Using Balanced Unequal Clustering Technique.' Pervasive Computing: A Networking Perspective and Future Directions: 81-91.

17. Yang, X.S., 2010. Nature-inspired metaheuristic algorithms. Luniver press.

18. Shah-Hosseini, H., 2011. Principal components analysis by the galaxy-based search algorithm: a novel metaheuristic for continuous optimisation. International Journal of Computational Science and Engineering, 6(1-2), pp.132-140.

19. Eesa, A.S., Brifcani, A.M.A. and Orman, Z., 2013. Cuttlefish algorithm-a novel bio-inspired optimization algorithm. Internationa Journal of Scientific \& Engineering Research, 4(9), pp.1978-1986.

20. Kaveh, A., and Mahdavi, V.R., 2014. Colliding bodies optimization: a novel meta-heuristic method. Computers \& Structures, 139, pp.18-27.

21. Heidari, A.A., Mirjalili, S., Faris, H., Aljarah, I., Mafarja, M. and Chen, H., 2019. Harris hawks optimization: Algorithm and applications. Future Generation Computer Systems, 97, pp.849-872.

22. Sharma, N., and Gupta, V., 2020, January. Meta-heuristic based optimization of WSNs energy and lifetime-A Survey. In 2020 10th International Conference on Cloud Computing, Data Science \& Engineering (Confluence) (pp. 369-374). IEEE.

23. Chawra, V.K. and Gupta, G.P., 2020. Salp: Metaheuristic-Based Clustering for Wireless Sensor Networks. In Nature-Inspired Computing Applications in Advanced Communication Networks (pp. 41-56). IGI Global.

24. Hudson, J., Denzinger, J., Kasinger, H. and Bauer, B., 2011, October. Dependable risk-aware efficiency improvement for self-organizing emergent systems. In 2011 IEEE Fifth International Conference on Self-Adaptive and Self-Organizing Systems (pp. 11-20). IEEE.

25. Cowling, P., Kendall, G. and Han, L., 2002, May. An investigation of a hyperheuristic genetic algorithm applied to a trainer scheduling problem. In Proceedings of the 2002 Congress on Evolutionary Computation. CEC'02 (Cat. No. 02TH8600) (Vol. 2, pp. 1185-1190). IEEE.

26. E. K. Burke, E. Hart, G. Kendall, J. Newall, P. Ross, and S.,2003. Schulenburg. Hyper-heuristics:2003, An emerging direction in modern search technology. In F. Glover and G. Kochenberger, editors, Handbook of Metaheuristics, pages 457-474. Kluwer.

27. E. K. Burke, S. Petrovic, and R. Qu.2006. Case-based heuristic selection for timetabling problems. Journal of Scheduling, 9(2):115-132.

28. R. Qu and E. K. Burke. 2019; Hybridisations within a graph-based hyper-heuristic framework for university timetabling problems. Journal of the Operational Research Society, 60:1273-1285, 2019.

29. E. K. Burke, M. R. Hyde, G. Kendall, and J. 2010.; Woodward. A genetic programming Hyper-heuristic approach for evolving two-dimensional strip packing heuristics. IEEE Transactions on Evolutionary Computation.

30. E. K. Burke, E. Hart, G. Kendall, J. Newall, P. Ross, and S.2003, Schulenburg. Hyper-heuristics: An emerging direction in modern search technology. In F. Glover and G. Kochenberger, editors, Handbook of Metaheuristics, pages 457-474. Kluwer.

31. Tsai, C.W., Chang, W.L., Hu, K.C. and Chiang, M.C., 2017. An improved hyper-heuristic clustering algorithm for wireless sensor networks. Mobile Networks and Applications, 22(5), pp.943-958.

32. Cai X, Wang P, Du L, Cui Z, Zhang W, Chen J.2019., Multi-objective three-dimensional DV-hop localization algorithm with NSGA-II. IEEE 19(21):10003-10015.

33. Wang, X.F. and Chen, G., 2002. Synchronization in small-world dynamical networks. International Journal of Bifurcation and chaos, 12(01), pp.187-192.

34. Wang, J., Gao, Y., Liu, W., Sangaiah, A.K. and Kim, H.J., 2019. An improved routing schema with special clustering using PSO algorithm for heterogeneous wireless sensor network. Sensors, 19(3), p.671.

35. Rai, M., Verma, S. and Tapaswi, S., 2009, March. A heuristic for minimum connected dominating set with local repair for wireless sensor networks. In 2009 Eighth International Conference on Networks (pp. 106-111). IEEE.

Published By:

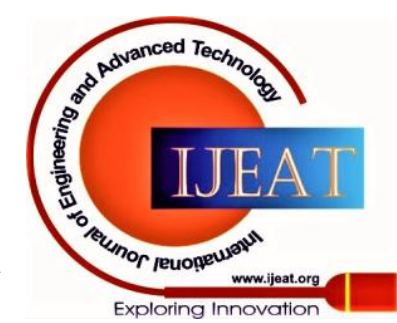


36. Tsai, C.W., Liu, S.J., and Wang, Y.C., 2018. A parallel metaheuristic data clustering framework for cloud. Journal of Parallel and Distributed Computing, 116, pp.39-49.

37. Zlobinsky, N. and Cheng, L., 2018. SAM: A Meta-Heuristic Algorithm for Single machine Scheduling Problems. SAIEE Africa Research Journal, 109(1), pp.58-68.

38. de León, A.D, Lalla-Ruiz, E. Melián-Batista, B. Moreno-Vega, J.M 2017; A Machine Learning-based system for berth scheduling at bulk terminals. Expert Syst. Appl. 2017, 87, 170-182.

39. Garcia, G.T., Sanchez, V.M., Marin, C.N.L., Cortez, J.I., Acevedo, C.A.R., Gonzalez, G.S.A., Ameca, J.L.H. and Garcia, M.D.C.M. 2018. Wireless sensor network for monitoring physical variables applied to green technology (IoT green technology). European Journal of Electrical Engineering and Computer Science, 2(2).

40. Sayed, G.I.; Tharwat, A.; Hassanien, A.E.2019; Chaotic dragonfly algorithm: An improved metaheuristic algorithm for feature selection. Appl. Intell. 49, 188-205.

41. Chou, J.S.; Nguyen, T.K. 2018., Forward Forecast of Stock Price Using Sliding-Window Metaheuristic-Optimized Machine-Learning Regression. IEEE Trans. Ind. Inf. 2018, 14, 3132-3142.

42. Wang, J., Gao, Y., Liu, W., Sangaiah, A.K., and Kim, H.J., 2019. An improved routing schema with special clustering using the PSO algorithm for heterogeneous wireless sensor networks. Sensors, 19(3), p.671.

43. Yin, L.; Luo, J.; Luo, H. 2018, Tasks Scheduling and Resource Allocation in Fog Computing Based on Containers for Smart Manufacturing. IEEE Trans. Ind. Inform. 2018, 14, 4712-4721.

44. García, J., Moraga, P., Valenzuela, M. and Pinto, H., 2020. A db-Scan Hybrid Algorithm: An Application to the Multidimensional Knapsack Problem. Mathematics, 8(4), p.507.

45. Hoanga, N.D., Nguyenb, H.T., Đức, H.N. and Thành, N.H., Differential Evolution with $\varepsilon$ constrained handling method developed in Excel VBA for solving optimization problem in civil engineering.

46. Kumar, A., Khosla, A., Saini, J.S., and Singh, S., 2012, June. Meta-heuristic range-based node localization algorithm for wireless sensor networks. In 2012 International Conference on Localization and GNSS (pp. 1-7). IEEE.

47. Mann, P.S., and Singh, S., 2017. Improved meta-heuristic based energy-efficient clustering protocol for wireless sensor networks. Engineering Applications of Artificial Intelligence, 57, pp.142-152.

48. Krishna, M.B., and Doja, M.N., 2015. Multi-objective meta-heuristic approach for energy-efficient secure data aggregation in wireless sensor networks. Wireless Personal Communications, 81(1), pp.1-16.

49. Agudo, J.E., Valenzuela-Valdés, J.F., Luna, F., Luque-Baena, R.M., and Padilla, P., 2016. Analysis of beamforming for improving the energy efficiency in wireless sensor networks with metaheuristics. Progress in Artificial Intelligence, 5(3), pp.199-206.

50. Mihoubi, M., Rahmoun, A., and Lorenz, P., 2017, October. Metaheuristic RSSI based on node localization in distributed wireless sensor networks. In 2017 Global Information Infrastructure and Networking Symposium (GIIS) (pp. 64-70). IEEE.

51. Ali, H., Tariq, U.U., Hussain, M., Lu, L., Panneerselvam, J. and Zhai, X., 2020. ARSH-FATI a Novel Metaheuristic for Cluster Head Selection in Wireless Sensor Networks. IEEE Systems Journal.

52. Gu, Z., Wang, Y., Shen, T. and Lau, F.C., 2020. On Heterogeneous Sensing Capability for Distributed Rendezvous in Cognitive Radio Networks. IEEE Transactions on Mobile Computing.

53. Yari, M., Hadikhani, P. and Asgharzadeh, Z., 2020. Energy-efficient topology to enhance the wireless sensor network lifetime using connectivity control. arXiv preprint arXiv:2005.03370.

54. Shankar, T., Karthikeyan, A., Sivasankar, P., and Rajesh, A., 2017. A hybrid approach for optimal cluster head selection in WSN using leach and monkey search algorithms. Journal of Engineering Science and Technology, 12(2), pp.506-517.

55. Wang P, Huang J, Cui Z, Xie L, Chen J. 2019; A Gaussian error correction multi-objective positioning model with NSGA-II. Concurrency Computat Pract Exper. 2019.

56. Wang P, Xue F, Li H, Cui Z, Xie L, Chen J. A, 2019 multi-objective DV-hop localization algorithm based on NSGA-II in the Internet of Things. Mathematics. 2019;7(2):184.

57. Sharmila, R., and Vijayalakshmi, V., 2015. Hybrid key management scheme for wireless sensor networks. International Journal of Security and Its Applications, 9(11), pp.125-132.

58. Kaur, S., and Mahajan, R., 2018. Hybrid meta-heuristic optimization-based energy efficient protocol for wireless sensor networks. Egyptian Informatics Journal, 19(3), pp.145-150.
59. Mehta, D., and Saxena, S., 2020. MCH-EOR: Multi-objective Cluster Head based Energy-aware Optimized Routing Algorithm in Wireless Sensor Networks. Sustainable Computing: Informatics and Systems, p.100406.

60. Chen, C.P., Mukhopadhyay, S.C., Chuang, C.L., Lin, T.S., Liao, M.S., Wang, Y.C., and Jiang, J.A., 2014. A hybrid memetic framework for coverage optimization in wireless sensor networks. IEEE Transactions on Cybernetics, 45(10), pp.2309-2322.

61. Tam, W. W., Tang, A., Woo, B., and Goh, S.Y., 2019. Perception of the Preferred Reporting Items for Systematic Reviews and Meta-Analyses (PRISMA) statement of authors publishing reviews in nursing journals: a cross-sectional online survey. BMJ Open, 9 (4): p.e026271.

62. Moher, David, Alessandro Liberati, Jennifer Tetzlaff, and Douglas G. Altman., 2010. "Preferred reporting items for systematic reviews and meta-analyses: the PRISMA statement." Int J Surg 8, no. 5: 336-341.

63. Page, M.J., McKenzie, J.E., Bossuyt, P.M., Boutron, I., Hoffmann, T. Mulrow, C.D., Shamseer, L. and Moher, D., 2020. Mapping of reporting guidelines for systematic reviews and meta-analyses generated a comprehensive item bank for future reporting guidelines. Journal of clinical epidemiology, 118, pp.60-68.

64. Munn, Zachary, Sandeep Moola, Dagmara Riitano, and Karolina Lisy., 2014. "The development of a critical appraisal tool for use in systematic reviews addressing questions of prevalence." International journal of health policy and management 3, no. 3, 123.

65. Fabrizi, F., Cerutti, R., Donato, F.M., and Messa, P., 2020. HBV infection is a risk factor for chronic kidney disease: Systematic review and meta-analysis. Revista Clinica Espanola.

\section{AUTHORS PROFILE}

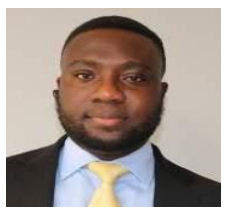

Oluwasegun. J. Aroba, A PhD Student and lecturer in Durban University of Technology, Department of Information Systems. A graduate of Information Technology University from prestigious Coventry University United Kingdom, BSc. Computer Science and Technology (Upper Class Division) Crawford University Nigeria whose research inclination focus area are into Wireless Sensor Network, Hyper-Heuristic, Hybrid-Heuristic and Meta-Analysis, Project Management, SAP Specialist with a decade years of experience in the healthcare industries, government parastatals, a consultant across the globe, graduate member of member of IEEE, Member of IET UK.

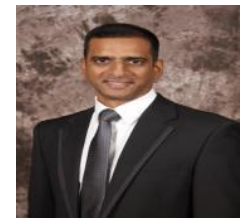

Dr. N. Naicker Education background is as follows: PhD [Information Systems \& Technology]; MSc [Information Systems]; Hons BSc (Computer Science); BSc (Computer Science). He currently serves as head of the Information Systems Department at the Durban University of Technology. He is currently involved with the supervision of $\mathrm{PhD}$ and Masters students at the Department of Information Systems; Information Technology and Computer Science. He is a member of the ICT and Society Research Group for the Faculty of Accounting and Informatics at Durban University of Technology.

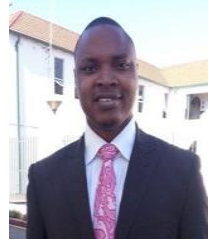

Dr. Timothy T. Adeliyi is a Lecturer in the Department of Information Technology at the Durban University of Technology. He received his $\mathrm{PhD}$ in Information Technology, MSc in Data Networks \& Security and BSc in Information Technology. His research inclinations are multimedia systems, machine learning, computer networks and security. He is a member of the ICT and Society Research Group of Durban University of Technology

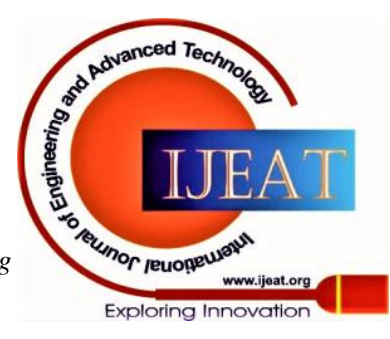


Meta-Analysis of Heuristic Approaches for Optimizing Node Localization and Energy Efficiency in Wireless Sensor Networks

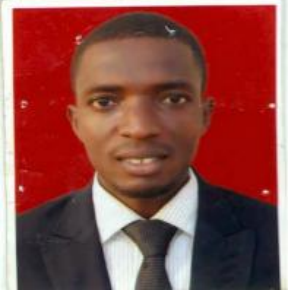

Dr. R. E. Ogunsakin is a statistician and postdoctoral fellow at Durban University of Technology, South Africa. His research interest is mostly based on statistical modeling and meta-analysis.

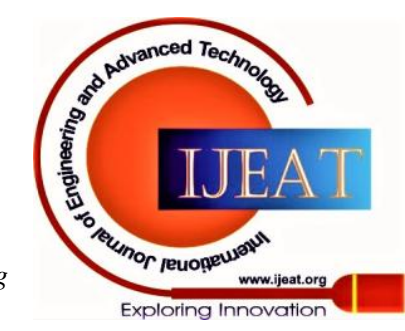

